\title{
UMA INTERFACE LAB-MADE PARA AQUISIÇÃO DE SINAIS ANALÓGICOS INSTRUMENTAIS VIA PORTA
} PARALELA DO MICROCOMPUTADOR

Edvaldo da Nóbrega Gaião, Everaldo Paulo de Medeiros, Wellington da Silva Lyra, Pablo Nogueira Teles Moreira, Edvan Cirino da Silva e Mário César Ugulino de Araújo*

Departamento de Química, Centro de Ciências Exatas e da Natureza, Universidade Federal da Paraíba, CP 5093, 58051-970 João Pessoa - PB

Recebido em 5/8/03; aceito em 20/1/04; publicado em 17/6/04

\begin{abstract}
A LAB-MADE INTERFACE FOR ACQUISITION OF INSTRUMENTAL ANALOG SIGNALS AT THE PARALLEL PORT OF A MICROCOMPUTER. A lab-made interface for acquisition of instrumental analog signals between 0 and $5 \mathrm{~V}$ at a frequency up to $670 \mathrm{kHz}$ at the parallel port of a microcomputer is described. Since it uses few and small components, it was built into the connector of a printer parallel cable. Its performance was evaluated by monitoring the signals of four different instruments and similar analytical curves were obtained with the interface and from readings from the instrument' displays. Because the components are cheap $(\sim \mathrm{U} \$ 35,00)$ and easy to get, the proposed interface is a simple and economical alternative for data acquisition in small laboratories for routine work, research and teaching.
\end{abstract}

Keywords: lab-made interface; data acquisition; instrumental analog signals.

\section{INTRODUÇÃO}

$\mathrm{Na}$ busca de resultados mais rápidos e confiáveis, o interfaceamento de instrumentos analíticos ao microcomputador é uma tendência natural em todos os laboratórios de análises de rotina e/ou pesquisa. Hoje em dia, os microcomputadores são comumente usados para controle, aquisição e processamento dos dados analíticos. Isto permite que uma maior quantidade de informações por unidade de tempo possam ser processadas com considerável confiabilidade, diminuindo o tempo e os custos das análises. A aquisição de sinais analógicos de instrumentos pode ser feita por meio de interfaces comerciais $^{1,2}$ ou por interfaces construídas no laboratório ${ }^{3-5}$. Entretanto, tais interfaces possuem custo relativamente alto e eletrônica complexa, o que dificulta a sua aquisição ou construção em laboratórios com poucos recursos e com pessoal pouco experiente em eletrônica digital e analógica.

Com o intuito de superar essas dificuldades é proposta, neste trabalho, uma interface simples e de baixo custo para a aquisição de sinais em equipamentos com saída analógica. A interface foi projetada de modo a usar poucos componentes eletrônicos e a ser construída no conector "macho" (DB25) de um cabo paralelo de impressora. Sendo assim, a interface é portátil, não necessita ser inserida dentro do microcomputador e pode ser usada em pesquisas de campo, pois é facilmente acoplada a microcomputadores "laptops" que possuam porta paralela EPP (“Enhanced Parallel Port”) 6.

\section{PARTE EXPERIMENTAL}

\section{A interface proposta}

A interface proposta neste trabalho usa apenas um conversor A/D de 8 bits (AD 0804), que é responsável pela conversão dos sinais analógicos em digitais e um circuito RC para controle do "timer". Qualquer software (Visual Basic, C, Pascal, etc) pode ser usado na

*e-mail: laqa@quimica.ufpb.br aquisição e arquivamento dos dados. Neste trabalho foi usado um programa escrito em Labview 5.1.

A interface proposta usa poucos e pequenos componentes eletrônicos, de modo que foi possível construí-la no conector "macho" (conector DB25) de um cabo de comunicação paralela (o cabo comum de uma impressora), como pode ser visto na Figura 1.

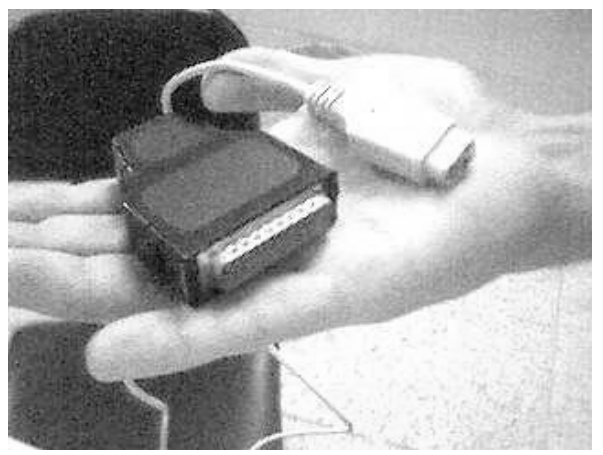

Figura 1. Interface proposta construída no conector de um cabo de impressora

O circuito eletrônico da interface proposta é simples (Figura 2) e pode ser construído por pessoas com pouca experiência em eletrônica. Possui os seguintes componentes: 2 pontas de provas, que podem ser dois fios, usados para conectar a interface às saídas analógicas dos instrumentos; 1 conector DB25 "macho", usado para acoplar a interface à porta paralela do microcomputador; 1 conversor analógico digital A/D (circuito integrado ADC0804) de 8 bits; 3 resistores e 1 capacitor.

$\mathrm{O}$ A/D é alimentado pelo seu pino 20 com uma tensão $+5 \mathrm{~V}$ que pode ser fornecida por uma fonte externa ou pela própria fonte de alimentação interna do microcomputador. Segundo especificação do fabricante do A/D (circuito integrado ADC0804), uma tensão de referência de aproximadamente $2,5 \mathrm{~V}$ deve ser aplicada no pino 9. Esta tensão foi gerada no circuito da interface proposta usando um divisor de tensão formado pelos resistores $\mathrm{R} 2(8,2 \mathrm{~K} \Omega)$ e R3 (8,2 K $\Omega$ ). 


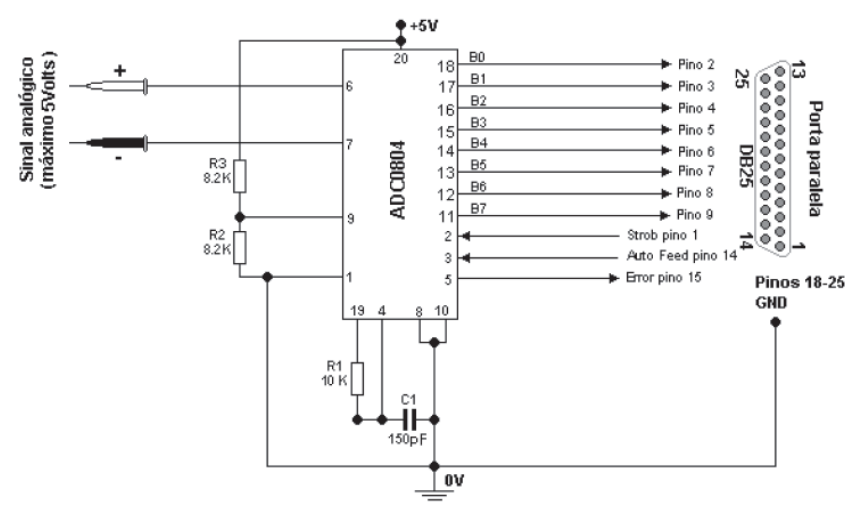

Figura 2. Circuito eletrônico da interface proposta

$\mathrm{O}$ A/D realiza as conversões de analógico para digital de sinais instrumentais analógicos de 0 a $5 \mathrm{~V}$ com resolução de 0,019 Volts, calculada pela relação $5 \mathrm{~V} / 2^{\mathrm{n}}$ (onde $\mathrm{n}=8$, que é o número de bits do conversor A/D), e com freqüência de $670 \mathrm{kHz}$. Esta frequiência é definida pelo circuito RC (Figura 2), formado pelo capacitor C1 $(150 \mathrm{pF})$ e o resistor R1 $(10 \mathrm{k} \Omega)$ e pode ser calculada por $\mathrm{f}=1 /(10 \mathrm{k} \Omega * 150 \mathrm{pF})$.

Após a conversão do sinal analógico, o sinal digital é disponibilizado pelo A/D nos pinos 11 a 18. Este sinal é lido pela porta paralela através dos pinos 2 a 9 . Para a aquisição dos sinais por meio da porta paralela é necessário inicialmente habilitar esta porta para o modo de comunicação EPP ("Enhanced Parallel Port") 6 no "setup" do microcomputador. No modo EPP, a porta paralela pode ser operada de forma bidirecional, ou seja, os dados podem ser enviados ou recebidos pelo microcomputador através dos pinos de dados 2 a 9 . Para evitar problemas de comunicação, a interface deve ser conectada ao microcomputador somente após habilitar a porta paralela no modo EPP.

Para dar início à aquisição dos sinais é necessário habilitar a porta paralela no modo de aquisição e enviar um sinal baixo nos pinos 2 e 3 do A/D. Isto é feito simplesmente enviando uma palavra (um "byte") de controle igual "23" (em hexadecimal) ao endereço 37A (em hexadecimal) do registrador de controle da porta paralela. Após o envio deste "byte" de controle, o A/D realiza a conversão e disponibiliza o sinal digital nos seus pinos de 11 a 18, que é lido nos pinos do registrador de dados da porta paralela cujo endereço é 378 (em hexadecimal). Após a leitura, um "byte" de controle igual a 20 (em hexadecimal) deve ser enviado ao endereço 37A do registrador de controle para mudar o "status" do AD e dar início a uma nova conversão. A rotina de aquisição de sinais empregando a interface proposta é mais facilmente compreendida usando o fluxograma apresentado na Figura 3.

\section{Utilização da interface proposta em análises instrumentais}

Para avaliar a performance da interface proposta, esta foi acoplada à saída analógica de quatro instrumentos analíticos diferentes: um fotocolorímetro Jenway, modelo 6061; um fotômetro de chama Digimed, modelo NK 2004; um condutivímetro Metrohm, modelo 712, e um pHmetro Metrohm, modelo 713. Para fins de comparações, diferentes curvas analíticas foram construídas usando as medidas feitas com a interface proposta e as medidas realizadas diretamente nos "displays" digitais dos instrumentos. Com o fotocolorímetro foram construídas curvas analíticas para determinação de íon ferroso usando o método do reagente colorimétrico 1,10-fenantrolina. Com o fotômetro de chama foram construídas as curvas analíticas para a

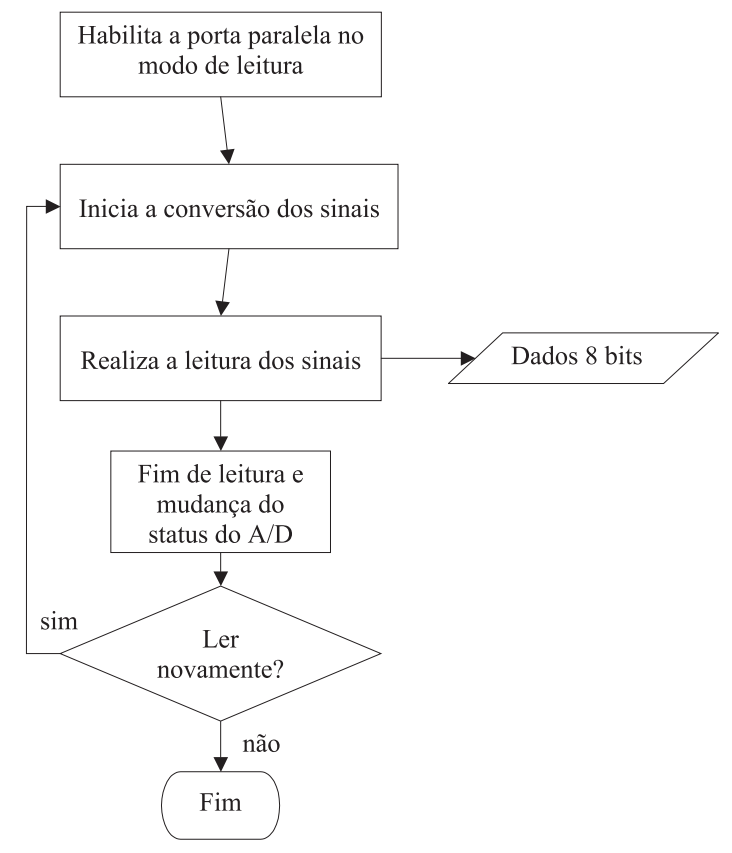

Figura 3. Diagrama de fluxo do procedimento de aquisição de sinais analógicos pela interface

determinação de potássio. O condutivímetro foi usado na construção de curvas de titulação de misturas de ácidos $\left(\mathrm{HCl}\right.$ e $\left.\mathrm{CH}_{3} \mathrm{COOH}\right)$ e o potenciômetro foi usado na construção das curvas de titulação para determinação de acidez total em vinhos.

$\mathrm{Na}$ construção de todas as curvas analíticas foram utilizados os métodos comumente encontrados na literatura usual de Química Analítica Instrumental ${ }^{7}$. Todas as soluções foram preparadas utilizando água recém destilada e deionizada e todos os reagentes usados foram de grau analítico.

\section{RESULTADOS E DISCUSSÃO}

As curvas analíticas obtidas usando as medidas feitas com a interface proposta e usando as leituras realizadas diretamente nos "displays" digitais dos quatro instrumentos interfaceados são mostradas na Figura 4.

As curvas da Figura 4 apresentam os mesmos valores numéricos no eixo das ordenadas pelos motivos explicados a seguir. Nas medidas com o fotocolorímetro, com condutivímetro ou com fotômetro de chama, os instrumentos geravam em seus "displays" valores numéricos entre 0,000 a 2,000 em unidades de absorbância, condutância ou intensidade. Idênticos valores numéricos entre 0,000 a 2.000 em $\mathrm{mV}$ eram também disponibilizados na saída analógica dos instrumentos. Estes sinais analógicos em $\mathrm{mV}$ eram digitalizados pela interface e arquivados no microcomputador como valores correspondentes a 0,000 a 2,000 em unidades de absorbância, condutância ou intensidade. Nas medidas com o pHmetro, a saída analógica do instrumento foi ajustada de modo que um sinal de 0,00 a $14,00 \mathrm{pH}$ no "display" produzia em sua saída analógica uma tensão de 0,00 a $2.000 \mathrm{mV}$. Assim, um valor de $\mathrm{pH}=1,00$ no "display" do pHmetro gerava um sinal de 142,9 mV na saída analógica. O software desenvolvido para aquisição do sinal analógico do pHmetro pela interface proposta, ao digitalizar um sinal de $142,9 \mathrm{mV}$ da saída analógica do pHmetro arquivava este sinal como correspondente a um valor de $\mathrm{pH}=1,00$.

Observa-se na Figura 4 que não existem diferenças significativas entre as curvas analíticas obtidas através de ambos os procedimentos 
a)

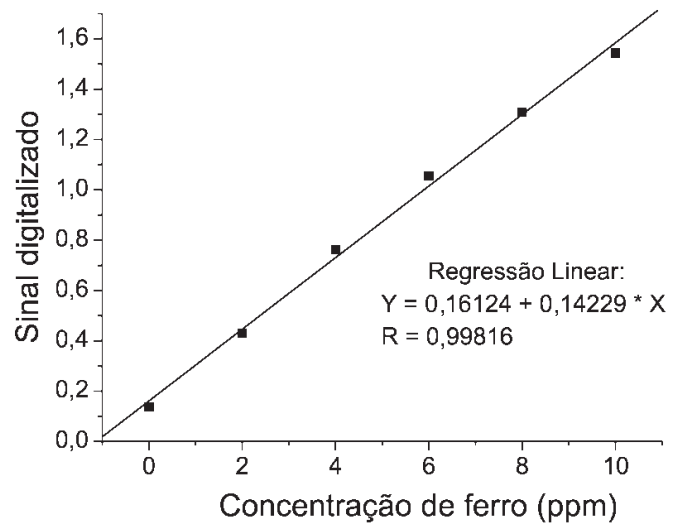

b)

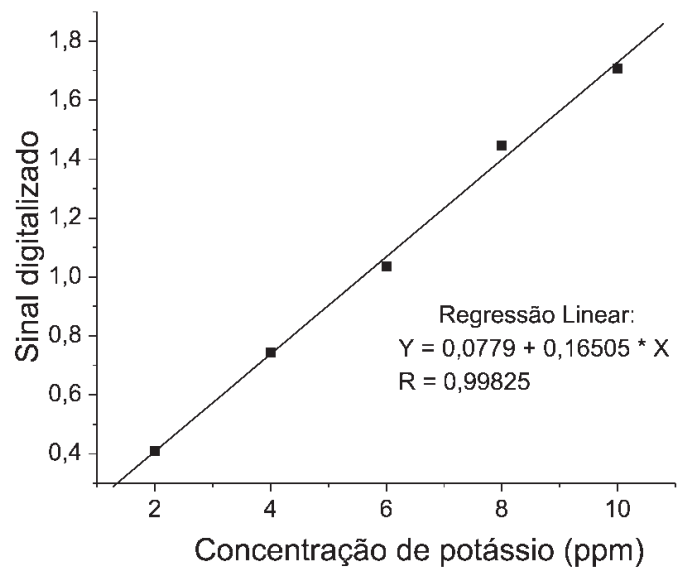

c)

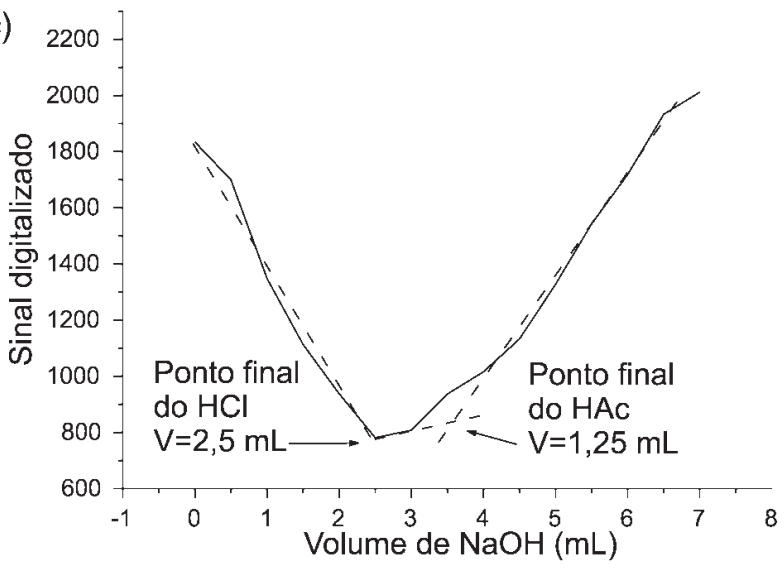

d)

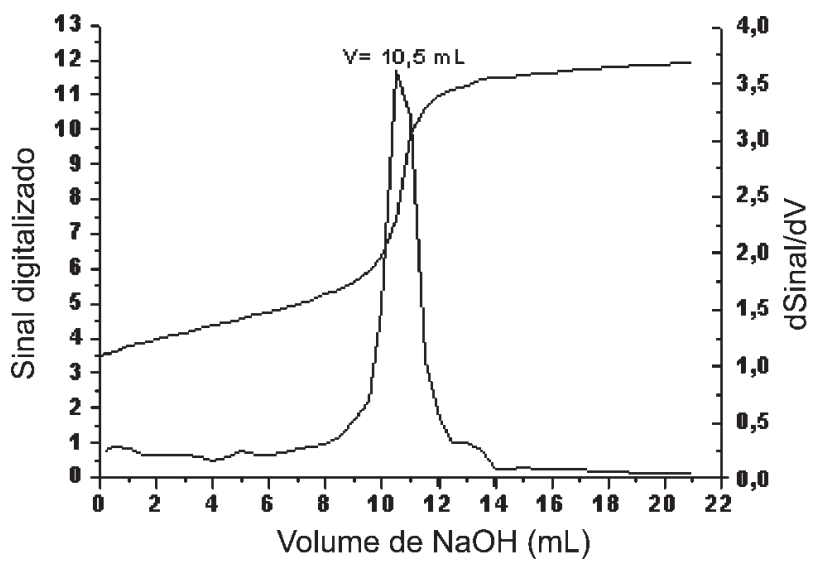

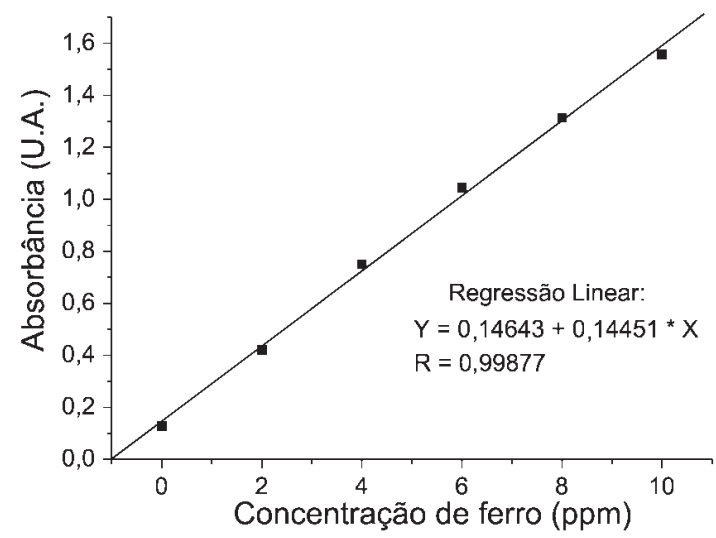
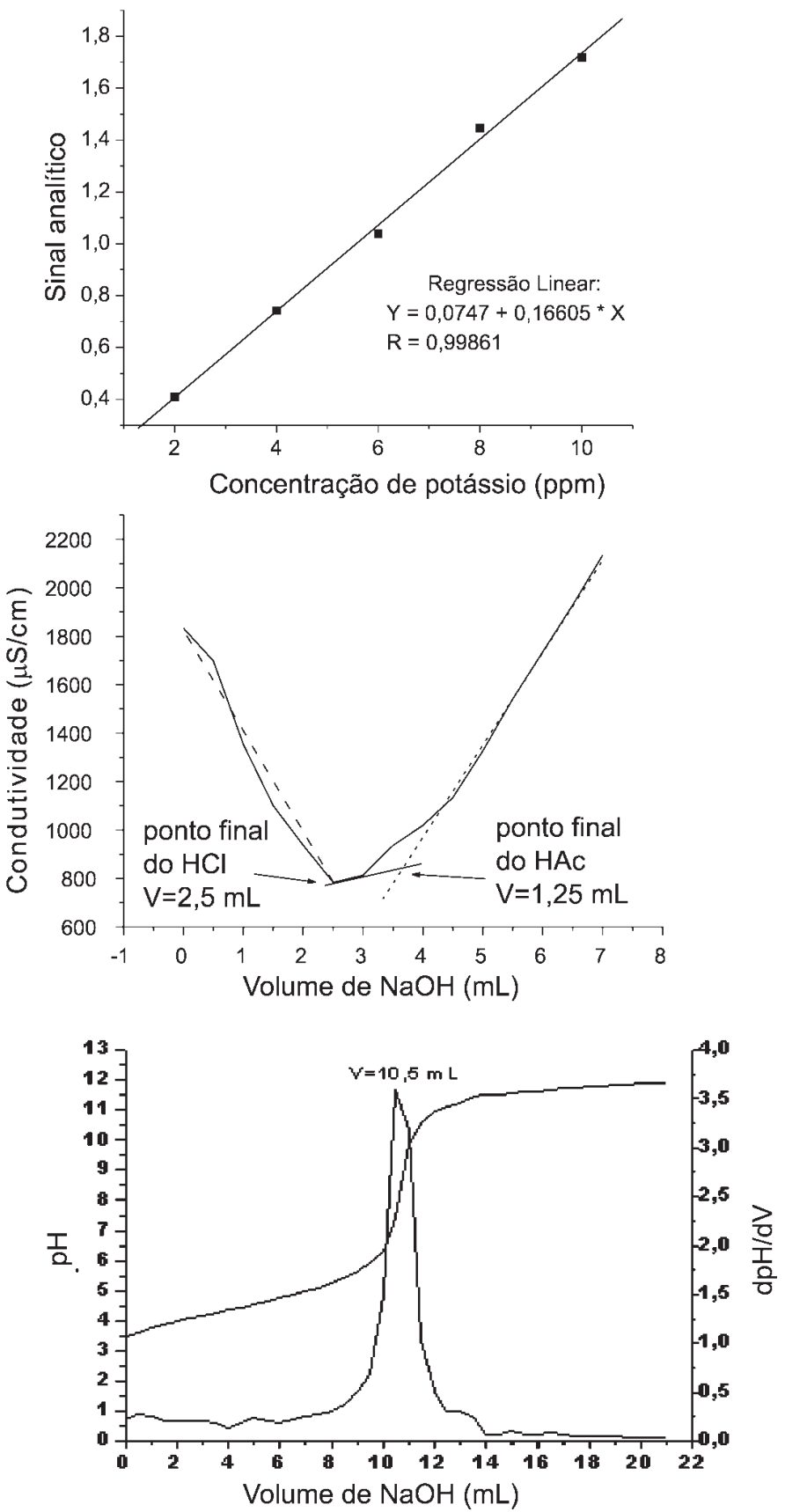

Figura 4. Curvas analíticas obtidas usando as medidas feitas com a interface proposta (curvas à esquerda) e as leituras realizadas diretamente nos displays digitais dos quatro instrumentos interfaceados (curvas à direita): $a=$ fotocolorímetro, $b=$ fotômetro de chama, $c=$ condutivímetro e $d=$ potenciômetro 
de leitura dos sinais. Por exemplo, os volumes de equivalência obtidos nas curvas de titulação condutimétrica e potenciométrica foram idênticos para as medidas realizadas via interface ou "displays" digitais.

Se o objetivo de um analista é apenas a digitalização de sinais analógicos instrumentais, a interface proposta pode ser considerada uma alternativa bastante atraente", devido ao seu baixo custo (cerca de U\$ 35,00$)$ e sua facilidade de construção. Para o mesmo propósito, a compra de uma interface comercial que normalmente contém conversores A/D e D/A, além de entrada e saída de sinais digitais, torna-se desnecessária, principalmente se levarmos em conta seu custo de aquisição (cerca de U\$ 400,00).

Uma alternativa de mais baixo custo que a interface comercial é usar multímetros digitais interfaceáveis a um microcomputador para a aquisição de dados de equipamentos com saída analógica. Estes multímetros são facilmente adquiríveis no mercado nacional e têm a importante vantagem de serem acompanhados do software de aquisição de dados. Entretanto, o preço de um multímetro deste tipo é de cerca de U\$ 240.00 e a freqüência de aquisição de sinais é muito baixa ( 2 pontos por segundo), quando comparada com a interface proposta, cuja frequiência é de até 670.000 pontos por segundo.

\section{CONCLUSÕES}

A interface proposta neste trabalho para aquisição de sinais instrumentais através da porta paralela de um microcomputador apre- sentou um desempenho bastante satisfatório quando acoplada a quatro diferentes instrumentos analíticos. Por usar poucos componentes, a interface pode ser construída de modo compacto no conector "macho" (DB25) de um cabo paralelo de impressora. Vale ressaltar que seus componentes são de fácil aquisição. Uma outra característica importante desta interface é não necessitar de instalação interna nos microcomputadores, o que não cria problemas de perda de garantia e ainda possibilita a sua fácil adaptação a microcomputadores "laptops", usados em pesquisas de campo. Todas estas características apresentadas fazem da interface proposta uma alternativa prática e econômica para a digitalização de sinais analíticos em laboratórios de análises de rotina, de pesquisa e/ou ensino com pouco recurso financeiro.

\section{REFERÊNCIAS}

1. http://www.advantech.com, acessada em Julho 2003.

2. http://www.natinst.com, acessada em Julho 2003.

3. Bellato, C. R.; Rohwedder, J. J. R.; Raimundo, I. M.; Pasquini, C.; J. Autom. Chem. 1996, 18, 7.

4. Martins, V. L.; Moreira, P. N. T.; Almeida, L. F.; Gaião, E. N.; Silva, E. C.; Angnes, L.; Araujo, M. C. U.; Controle \& Instrumentação 2002, 7, 80.

5. Lago, C. L. do; Quim. Nova 1993, 16, 237.

6. Torres, G.; Hardware Curso Completo, $4^{\text {th }}$ ed., Axcel Books: Rio de Janeiro, 2001.

7. Skoog, D. A.; West, D. M.; Holler, F. J.; Fundamentals of Analytical Chemistry, $6^{\text {th }}$ ed., Saunders College Publishing: New York, 1992. 